\title{
Global uniform estimate for the modulus of $2 D$ Ginzburg-Landau vortexless solutions with asymptotically infinite boundary energy
}

\author{
Radu Ignat* $\quad$ Matthias Kurzke ${ }^{\dagger} \quad$ Xavier Lamy ${ }^{\ddagger}$
}

November 13, 2019

\begin{abstract}
For $\varepsilon>0$, let $u_{\varepsilon}: \Omega \rightarrow \mathbb{R}^{2}$ be a solution of the Ginzburg-Landau system

$$
-\Delta u_{\varepsilon}=\frac{1}{\varepsilon^{2}} u_{\varepsilon}\left(1-\left|u_{\varepsilon}\right|^{2}\right)
$$

in a Lipschitz bounded domain $\Omega$. In an energy regime that excludes interior vortices, we prove that $1-\left|u_{\varepsilon}\right|$ is uniformly estimated by a positive power of $\varepsilon$ globally in $\Omega$ provided that the energy of $u_{\varepsilon}$ at the boundary $\partial \Omega$ does not grow faster than $\varepsilon^{-\alpha}$ with $\alpha \in(0,1)$.
\end{abstract}

\section{Introduction}

Let $\Omega \subset \mathbb{R}^{2}$ be a Lipschitz bounded open connected set (not necessarily simply connected) with the unit outer normal and tangent vector fields $(\nu, \tau)$ defined a.e. on $\partial \Omega$ with

$$
\tau=\nu^{\perp}=\left(-\nu_{2}, \nu_{1}\right)
$$

so that $(\nu, \tau)$ forms an oriented frame a.e. on $\partial \Omega$. For every small $\varepsilon>0$, let $u_{\varepsilon}: \Omega \rightarrow \mathbb{R}^{2}$ be a solution of the Ginzburg-Landau system:

$$
\left\{\begin{aligned}
-\Delta u_{\varepsilon} & =\frac{1}{\varepsilon^{2}} u_{\varepsilon}\left(1-\left|u_{\varepsilon}\right|^{2}\right) & & \text { in } \Omega \\
u_{\varepsilon} & =g_{\varepsilon} & & \text { on } \partial \Omega
\end{aligned}\right.
$$

with the boundary data $g_{\varepsilon}: \partial \Omega \rightarrow \mathbb{R}^{2}$. For the boundary energy

$$
N_{\varepsilon}:=\int_{\partial \Omega} \frac{1}{2}\left|\partial_{\tau} g_{\varepsilon}\right|^{2}+\frac{1}{4 \varepsilon^{2}}\left(1-\left|g_{\varepsilon}\right|^{2}\right)^{2} d \mathcal{H}^{1}
$$

and the interior energy

$$
M_{\varepsilon}:=\int_{\Omega} \frac{1}{2}\left|\nabla u_{\varepsilon}\right|^{2}+\frac{1}{4 \varepsilon^{2}}\left(1-\left|u_{\varepsilon}\right|^{2}\right)^{2} d x,
$$

\footnotetext{
*Institut de Mathématiques de Toulouse \& Institut Universitaire de France, UMR 5219, Université de Toulouse, CNRS, UPS IMT, F-31062 Toulouse Cedex 9, France. Email: Radu.Ignat@math.univ-toulouse.fr

${ }^{\dagger}$ School of Mathematical Sciences, University of Nottingham, Nottingham NG7 2RD, United Kingdom. Email: matthias.kurzke@nottingham.ac.uk

‡Institut de Mathématiques de Toulouse, UMR 5219, Université de Toulouse, CNRS, UPS IMT, F-31062 Toulouse Cedex 9, France. Email: Xavier.Lamy@math.univ-toulouse.fr
} 
we assume that there exists a power $\alpha \in(0,1)$ such that 1

$$
M_{\varepsilon} \leq \kappa|\log \varepsilon| \quad \text { and } \quad N_{\varepsilon} \ll \frac{1}{\varepsilon^{\alpha}} \quad \text { as } \varepsilon \rightarrow 0,
$$

for some small constant $\kappa>0$ depending on the Lipschitz regularity of $\Omega$. The first condition in (4) avoids nucleation of interior vortices of non-vanishing winding number (because the energetic cost of an interior vortex of non-zero winding number is of order $|\log \varepsilon|$, see the seminal book of Bethuel-Brezis-Hélein (4)). The second condition in (4) corresponds to an energetic regime avoiding the presence of boundary vortices: indeed, a transition of $g_{\varepsilon}$ between two opposite directions at the boundary on a distance $\varepsilon$ (the length scale of a vortex) has an energetic cost of order $\frac{1}{\varepsilon}$ (see Example 1 below). If $N_{\varepsilon} \lesssim \frac{1}{\varepsilon}$, then solutions $u_{\varepsilon}$ of (1) may have zeros on the boundary (see Proposition 3).

\subsection{Main result}

Our main result is the following global uniform estimate in the regime (4) for the convergence of $\left|u_{\varepsilon}\right|$ to 1 in $\Omega$, which means that $1-\left|u_{\varepsilon}\right|$ behaves as a positive power of $\varepsilon$.

Theorem 1 Let $\Omega \subset \mathbb{R}^{2}$ be a Lipschitz bounded domain. There exists a (small) constant $\kappa>0$ depending on the Lipschitz regularity of $\Omega$ such that for every solution $u_{\varepsilon}$ of (1) satisfying (4) for some $\alpha \in(0,1)$ we have the following global estimate ${ }^{2}$

$$
\sup _{\Omega}|| u_{\varepsilon}|-1| \leq C\left(\varepsilon^{1-}\left(1+N_{\varepsilon}+M_{\varepsilon}\right)\left(1+M_{\varepsilon}\right)^{\frac{1}{2}-}\right)^{\frac{1}{6}-} \quad \text { as } \quad \varepsilon \rightarrow 0,
$$

for some constant $C>0$ depending only on the Lipschitz regularity ${ }^{3}$ of $\Omega$. In particular, $g_{\varepsilon}$ has zero winding number on $\partial \Omega$, i.e. 4

$$
\operatorname{deg}\left(g_{\varepsilon}, \partial \Omega\right):=\frac{1}{2 \pi} \int_{\partial \Omega} \frac{g_{\varepsilon}^{\perp}}{\left|g_{\varepsilon}\right|} \cdot \partial_{\tau}\left(\frac{g_{\varepsilon}}{\left|g_{\varepsilon}\right|}\right) d \mathcal{H}^{1}=0 .
$$

In particular, under the assumption of Theorem 1, we deduce that

$$
\sup _{\Omega}|| u_{\varepsilon}|-1| \leq C \varepsilon^{\frac{1-\alpha}{6}-} \quad \text { as } \quad \varepsilon \rightarrow 0 .
$$

We believe that the power $\frac{1}{6}-$ of $\varepsilon$ in the above estimate is not optimal; moreover, the optimal power of $\varepsilon$ is expected to be $\leq \frac{1}{2}$ (see $[9$ below). The proof of Theorem 1 is done in several steps. In Section 2, we obtain a preliminary estimate of the uniform convergence of $\left|u_{\varepsilon}\right|$ to 1 , but at a much slower rate than the one claimed in Theorem 1 . Thanks to this preliminary estimate, in Section 3, we will be able to use more efficiently the Ginzburg-Landau system (1) to deduce an improved rate for the convergence of $\left|u_{\varepsilon}\right|$ to 1 , first in the $L^{2}$-norm and then in the $L^{\infty}$-norm.

Remark 1 Our proof adapts with minor modifications to critical points of the energy

$$
E_{\varepsilon}\left(u_{\varepsilon} ; \Omega\right):=\int_{\Omega} \frac{1}{2}\left|\nabla u_{\varepsilon}\right|^{2}+\frac{1}{4 \varepsilon^{2}} F\left(|u|^{2}\right) d x,
$$

where $F \in C^{2}([0, \infty))$ satisfies $F \geq 0, F(1)=0$ and $(s-1) F^{\prime}(s) \geq c(1-s)^{2}$ for all $s \geq 0$ and some constant $c>0$. The typical example is $F(s)=(1-s)^{2}$.

\footnotetext{
${ }^{1}$ We write $a \ll b$ if $\frac{a}{b} \rightarrow 0$, and $a \lesssim b$ if $\frac{a}{b}$ is bounded by a universal constant.

${ }^{2}$ We denote by $a+$ (resp. $a-$ ) any number strictly bigger than $a$ (resp. strictly smaller than $a$ ) that one can think of as close to $a$. The constants in inequalities involving $a+$ or $a$ - may depend on the choice of these numbers.

${ }^{3}$ In fact, $C>0$ depends only on the lowest angle and lowest radius of interior and exterior cones at any point of the Lipschitz boundary $\partial \Omega$.

${ }^{4}$ In general, $\partial \Omega$ is not connected; the definition of the degree is coherent with the choice of the orientation $\tau=\nu^{\perp}$ given by the outer normal field $\nu$.
} 


\subsection{Optimality of our assumptions}

Let us discuss the optimality of the assumption (4) in Theorem 1. First, the assumption on $M_{\varepsilon}$ in (4) is optimal: if the constant $\kappa$ is not small enough, then solutions $u_{\varepsilon}$ of (1) may vanish inside $\Omega$. Moreover, the threshold value of $\kappa$ at which this happens can be arbitrarily small depending on the Lipschitz regularity of the domain:

Proposition 2 For any $\theta_{0} \in(0, \pi)$ and any $\eta>0$ there exists a cone shape domain $\Omega$ of opening angle $\theta_{0}$, an exponent $\alpha \in(0,1)$ and a solution $u_{\varepsilon}$ of the Ginzburg-Landau system (1) such that for small $\varepsilon>0, u_{\varepsilon}\left(P_{\varepsilon}\right)=0$ for a degree-one vortex point $P_{\varepsilon} \in \Omega$ and (4) holds true for $\kappa=\frac{\theta_{0}}{2}+\eta$.

Second, the assumption on $N_{\varepsilon}$ in (4) is near-optimal in the following sense: if $N_{\varepsilon} \lesssim \frac{1}{\varepsilon}$, then a solution $u_{\varepsilon}$ of (1) may have zeros at the boundary of any Lipschitz domain $\Omega$.

Proposition 3 For any Lipschitz domain $\Omega$, there exists a solution $u_{\varepsilon}$ of the Ginzburg-Landau system (1) such that for small $\varepsilon>0, u_{\varepsilon}\left(x_{0}\right)=0$ for some $x_{0} \in \partial \Omega$, while $M_{\varepsilon} \lesssim 1$ and $N_{\varepsilon} \lesssim \frac{1}{\varepsilon}$.

We also point out that solutions of (1) with $M_{\varepsilon} \leq \kappa|\log \varepsilon|$ with small $\kappa$ may present an interior vortex with non-zero degree if the boundary energy is of order $\frac{1}{\varepsilon}$.

Proposition 4 For any smooth simply connected domain $\Omega$ and any small $\eta=\eta(\varepsilon)>0$, there exists a solution $u_{\eta}$ of the Ginzburg-Landau system (1) such that $\left|u_{\eta}\right|=1$ on $\partial \Omega$ and $\operatorname{deg}\left(u_{\eta}, \partial \Omega\right)=$ 1 (in particular, $u_{\eta}$ has a vortex on non-vanishing degree in $\Omega$ ), while $M_{\varepsilon} \lesssim\left(\frac{\eta}{\varepsilon}\right)^{2}|\log \eta|$ and $N_{\varepsilon} \lesssim \frac{1}{\eta}$. In particular

- we can choose $\eta=\eta(\varepsilon)>0$ such that $M_{\varepsilon} \lesssim 1$ and $N_{\varepsilon} \lesssim \frac{|\log \varepsilon|^{1 / 2}}{\varepsilon}$;

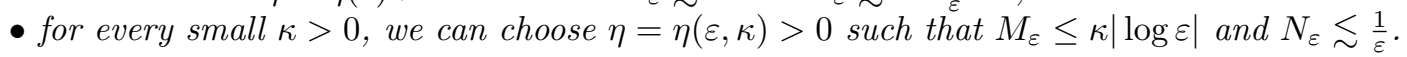

Finally we remark that even for $\mathbb{S}^{1}$-valued boundary data with zero degree, if $N_{\varepsilon} \gg \frac{1}{\varepsilon}$ then minimizers may have bounded energy but modulus not uniformly close to 1 . (This is related to Example 1 in Section 1.4 below.)

Proposition 5 For any smooth bounded domain $\Omega$ and $\eta(\varepsilon) \ll \varepsilon \ll 1$, there exists $g_{\varepsilon} \in H^{1}\left(\partial \Omega ; \mathbb{S}^{1}\right)$ with $\operatorname{deg}\left(g_{\varepsilon}, \partial \Omega\right)=0$ an $\oint^{5} N_{\varepsilon} \sim \frac{1}{\eta(\varepsilon)}$, such that any minimizer $u_{\varepsilon}$ of $E_{\varepsilon}(\cdot ; \Omega)$ under the Dirichlet boundary condition $u_{\varepsilon}=g_{\varepsilon}$ on $\partial \Omega$ satisfies

$$
\left.\sup _{\Omega}|1-| u_{\varepsilon}\right|^{2} \mid \geq \frac{1}{2} \quad \text { for } \varepsilon \ll 1,
$$

while $M_{\varepsilon} \lesssim 1$.

The proofs of Propositions 2 to 5 can be found in Section 4 . The case $N_{\varepsilon} \ll \frac{1}{\varepsilon}$ (i.e., $\alpha=1$ in the regime (4)) remains open; in that case, we conjecture that our global estimate in Theorem 1 should still hold true, at least in smooth domains.

\subsection{Related works}

There is a huge literature on the analysis of solutions $u_{\varepsilon}$ of the Ginzburg-Landau system (1). Let us only mention some of them (and apologize for omitting many other important ones).

In the seminal paper [3], Bethuel, Brezis and Hélein studied the system (1) on a smooth simply connected domain $\Omega$ for minimizers $u_{\varepsilon}$ of the associated energy functional, with a fixed smooth boundary data $g_{\varepsilon}:=g$ such that $|g|=1$ on $\partial \Omega$ and $g$ is of zero winding number (so $N_{\varepsilon}, M_{\varepsilon}$ are of

\footnotetext{
${ }^{5}$ We write $a \sim b$ if $a \lesssim b$ and $b \lesssim a$.
} 
order 1); they proved that ||$u_{\varepsilon}|-1|$ behaves as $\varepsilon^{2}$ globally in $\Omega$ and this rate is optimal. They also studied the case of non-fixed smooth boundary data $g_{\varepsilon}: \partial \Omega \rightarrow \mathbb{R}^{2}$ that is of zero winding number and has uniformly bounded energy $N_{\varepsilon} \lesssim 1$; then for minimizers $u_{\varepsilon}$, they deduced that $M_{\varepsilon} \lesssim 1$ and ||$u_{\varepsilon}|-1|$ behaves as $\varepsilon^{2}$ locally in $\Omega$. These results also hold for non-minimizing solutions if $u_{\varepsilon} \rightarrow u_{0}$ strongly in $H^{1}$ for some limit $u_{0}$, see [4, Remark A.1].

In [5, Bethuel, Orlandi and Smets considered solutions of (1) that need not be minimizing, without imposing any bounds on $M_{\varepsilon}$ or $N_{\varepsilon}$. They proved local estimates on ||$u_{\varepsilon}|-1|$, away from the boundary and from a vorticity set. In our setting, their results imply that ||$u_{\varepsilon}|-1|$ is of order at most $\varepsilon^{2(1-\beta)} M_{\varepsilon}$ in the region $\left\{x \in \Omega\right.$ : dist $\left.(x, \partial \Omega) \geq \varepsilon^{\beta}\right\}$, for any $\beta \in(0,1)$, but do not provide a good uniform estimate up to the boundary.

In the present work we focus on obtaining, for solutions of (1) that need not be minimizing, precise uniform estimates on ||$u_{\varepsilon}|-1|$ which hold:

- up to the boundary $\partial \Omega$ of a general Lipschitz domain,

- and in a regime that goes beyond the restrictive uniform bound $N_{\varepsilon} \lesssim 1$.

Estimates up to the boundary of a rectangle were obtained in [7, Appendix] in the regime $M_{\varepsilon}, N_{\varepsilon} \ll|\log \varepsilon|$. There it was proved that ||$u_{\varepsilon}|-1|$ is of order at most $\left(\frac{1+N_{\varepsilon}+M_{\varepsilon}}{|\log \varepsilon|}\right)^{\frac{1}{6}-}$ globally in $\Omega$. In Section 2 we will follow the same approach in a general Lipschitz domain and under the less restrictive regime (4), as a first step towards the stronger estimate of Theorem 1 .

\subsection{Motivation}

The energy functional $E_{\varepsilon}$ is a simplified version of a model describing superconducting materials. We simply mention here that ||$u_{\varepsilon}|-1|$ measures how close the system is to a superconducting state, and refer the interested reader to the monographs 4, 16.

Another motivation comes from several studies of the pattern formation in thin ferromagnetic films [11, 7, 10, where one wishes to approximate $u_{\varepsilon}$ by $\mathbb{S}^{1}$-valued maps away from the vortices. In a vortexless region $\Omega$ (assume e.g. $E_{\varepsilon}\left(u_{\varepsilon} ; \Omega\right) \ll|\log \varepsilon|$ ), the idea introduced in [11] consists in finding a (squared, spherical etc.) grid $\mathcal{R}_{\varepsilon}$, each cell of the grid having the size $\sim \varepsilon^{\beta}$ with $\beta \in(0,1)$ (i.e., much larger than the length-scale of a vortex) such that the energy $E_{\varepsilon}\left(u_{\varepsilon} ; \mathcal{R}_{\varepsilon}\right)$ on the 1-dimensional grid $\mathcal{R}_{\varepsilon}$ is of order $E_{\varepsilon}\left(u_{\varepsilon} ; \Omega\right) / \varepsilon^{\beta}$. Then Theorem 1 implies that ||$u_{\varepsilon}|-1|$ behaves as a positive power of $\varepsilon$ in $\Omega$, and $v_{\varepsilon}=u_{\varepsilon} /\left|u_{\varepsilon}\right|$ is a "good" approximation of $u_{\varepsilon}$ (in terms of the $L^{2}$ norm, their global Jacobian etc., see [10]). In that context, we give the following consequence of Theorem 1 for a cell of the grid leading to a key estimate needed in [10] (only a weaker version of this key estimate was needed in [11, 7]):

Corollary 6 Let $\mathcal{C} \subset \mathbb{R}^{2}$ be a Lipschitz bounded domain. Let $\varepsilon>0, \beta \in(0,1)$ and $\mathcal{C}_{\varepsilon}:=\varepsilon^{\beta} \mathcal{C}$ be a cell of size $\varepsilon^{\beta}$. Assume that $u_{\varepsilon}$ is a solution of (1) in $\mathcal{C}_{\varepsilon}$ with

$$
\int_{\partial \mathcal{C}_{\varepsilon}} \frac{1}{2}\left|\partial_{\tau} g_{\varepsilon}\right|^{2}+\frac{1}{4 \varepsilon^{2}}\left(1-\left|g_{\varepsilon}\right|^{2}\right)^{2} d \mathcal{H}^{1} \ll \frac{|\log \varepsilon|}{\varepsilon^{\beta}}
$$

and

$$
\int_{\mathcal{C}_{\varepsilon}} \frac{1}{2}\left|\nabla u_{\varepsilon}\right|^{2}+\frac{1}{4 \varepsilon^{2}}\left(1-\left|u_{\varepsilon}\right|^{2}\right)^{2} d x \ll|\log \varepsilon|,
$$

then

$$
|| u_{\varepsilon}|-1| \leq C \varepsilon^{\frac{1-\beta}{6}-} \quad \text { in } \quad \mathcal{C}_{\varepsilon}
$$

for some $C>0$ depending on the Lipschitz regularity of $\mathcal{C}$. In particular, $g_{\varepsilon}$ has zero winding number on $\mathcal{C}_{\varepsilon}$. 
Proof. Denoting the rescaled map $\tilde{u}_{\tilde{\varepsilon}}(\tilde{x}):=u_{\varepsilon}\left(\varepsilon^{\beta} \tilde{x}\right)$ for $\tilde{x} \in \mathcal{C}$ with $\tilde{\varepsilon}:=\varepsilon^{1-\beta}$, then $\tilde{u}_{\tilde{\varepsilon}}$ satisfies the system (1) with the parameter $\tilde{\varepsilon}$ instead of $\varepsilon$ and the boundary energy, resp. interior energy of $\tilde{u}_{\tilde{\varepsilon}}$ on $\partial \mathcal{C}$, resp. in $\mathcal{C}$ are estimated by $N_{\tilde{\varepsilon}}, M_{\tilde{\varepsilon}} \ll|\log \tilde{\varepsilon}|$. By Theorem 1 the conclusion follows.

As already hinted at, the regime (4) is motivated by the study of boundary vortices (see e.g. [14, 10]). In the absence of interior vortices, the first nontrivial example corresponds to a dipole of two compensating boundary vortices.

Example 1 Let $\Omega \subset \mathbb{R}^{2}$ be a bounded smooth domain containing the upper half unit ball, more precisely,

$$
\Omega \cap B(0,1)=\left\{x=\left(x_{1}, x_{2}\right) \in B(0,1): x_{2}>0\right\},
$$

where $B(0,1)$ is the unit ball centered at the origin. Let $\eta=\eta(\varepsilon) \in(0,1)$ be a parameter. Consider the boundary data $g_{\varepsilon}: \partial \Omega \rightarrow \mathbb{S}^{1}$ such that $g_{\varepsilon}(x)=e^{i \phi_{\varepsilon}}$ with

$$
\phi_{\varepsilon}(x)= \begin{cases}0 & \text { if } x \in \partial \Omega \backslash B(0, \eta), \\ \pi\left(1-\frac{\left|x_{1}\right|}{\eta}\right) & \text { if } x=\left(x_{1}, x_{2}\right) \in \partial \Omega \cap B(0, \eta) .\end{cases}
$$

(This is the prototype of a dipole of two boundary vortices corresponding of two consecutive transitions between opposite directions $\tau$ and $-\tau$ at the boundary at a distance $\eta)$. We extend $\phi_{\varepsilon}$ to the entire domain $\Omega$ by setting $\phi_{\varepsilon}=0$ in $\Omega \backslash B(0, \eta)$ and $\phi_{\varepsilon}(x)=\pi\left(1-\frac{|x|}{\eta}\right)$ if $x \in \Omega \cap B(0, \eta)$. Then we compute that

$$
N_{\varepsilon}=\int_{\partial \Omega} \frac{1}{2}\left|\partial_{\tau} g_{\varepsilon}\right|^{2} d \mathcal{H}^{1} \lesssim \frac{1}{\eta}, \quad E_{\varepsilon}\left(e^{i \phi_{\varepsilon}} ; \Omega\right) \lesssim 1
$$

Therefore, if $u_{\varepsilon}$ is a minimizer of $E_{\varepsilon}(\cdot ; \Omega)$ under the Dirichlet boundary condition $u_{\varepsilon}=g_{\varepsilon}$ on $\partial \Omega$, we have that $E_{\varepsilon}\left(u_{\varepsilon} ; \Omega\right) \leq E_{\varepsilon}\left(e^{i \phi_{\varepsilon}} ; \Omega\right)$ so that $(4)$ holds provided that $\frac{1}{\eta} \ll \frac{1}{\varepsilon^{\alpha}}$. In this case, Theorem 1 implies that $\left|u_{\varepsilon}\right|$ remains close to 1 as a positive power of $\varepsilon$, in particular, no interior vortices appear in $\Omega$. We highlight the fact that the regime $\varepsilon^{\alpha} \ll \eta$ with $\alpha \in(0,1)$ is essential in the above example for minimizers $u_{\varepsilon}$ to have modulus close to 1 uniformly. This scenario changes dramatically in the opposite regime $\eta \ll \varepsilon$ (see Proposition 5).

\section{Notations}

In the sequel we will use the symbol $\lesssim$ to denote an inequality up to a multiplicative constant that depends only on the Lipschitz regularity of $\Omega$, that is, on $\left(\rho_{0}, \theta_{0}\right) \in(0, \infty) \times(0, \pi)$ such that for all $x \in \partial \Omega$ there is a cone of vertex $x$, radius $\rho_{0}$ and opening angle $\theta_{0}$ which is included in $\bar{\Omega}$, and the opposite cone is included in $\mathbb{R}^{2} \backslash \Omega$ (this is the uniform cone property, see e.g. [8, Theorem 1.2.2.2]). We also note that, thanks to the uniform cone property, the rectangle

$$
R=\left(-\frac{\rho_{0}}{2} \sin \frac{\theta_{0}}{2}, \frac{\rho_{0}}{2} \sin \frac{\theta_{0}}{2}\right) \times\left(-\rho_{0} \cos \frac{\theta_{0}}{2}, \rho_{0} \cos \frac{\theta_{0}}{2}\right),
$$

has the following property: for all $x \in \Omega$, there exists an angle $\gamma=\gamma(x) \in \mathbb{R}$ such that for all $t \in(0,1]$, the set

$$
\mathcal{R}_{t}(x)=\left(x+t e^{i \gamma} R\right) \cap \Omega \text { is bi-Lipschitz homeomorphic to } t B,
$$

where $B$ is the unit ball, and the Lipschitz constants of the homeomorphism and its inverse are bounded by a constant depending only on $\left(\rho_{0}, \theta_{0}\right)$. See Figure 1 below. (The angle $\gamma$ just serves to rotate the rectangle in order to align it with the cone; Figure 1 corresponds to $\gamma=0$.)

We recall that for $a \in \mathbb{R}$ we write $a+($ resp. $a-)$ to denote any real number strictly greater (resp. smaller) than $a$ but that can be chosen arbitrarily close to $a$. In inequalities involving such exponents, the constant will also depend on the distance of that number to $a$.

We write $B(x, r)$ for the ball centered at $x$ of radius $r$. 
Figure 1: Cone property and rectangle $\mathcal{R}_{1}(x)$ at a boundary point $x \in \partial \Omega$

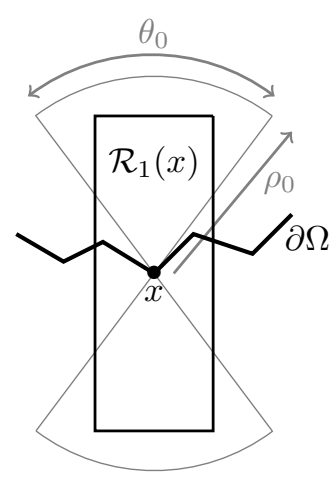

\section{$2 \quad$ A-priori global uniform estimate of $\left|u_{\varepsilon}\right|$ in $\Omega$}

The aim of this section is to prove the following weaker estimate of ||$u_{\varepsilon}|-1|$ in $\Omega$ :

Theorem 7 Let $\Omega \subset \mathbb{R}^{2}$ be a Lipschitz bounded domain. If $u_{\varepsilon}$ satisfies (1) and (4), then we have

$$
\sup _{\Omega}|| u_{\varepsilon}|-1| \lesssim\left(\frac{1+M_{\varepsilon}}{|\log \varepsilon|}\right)^{\frac{1}{6}-}
$$

In particular, if $\kappa$ is small enough in (4) then $\left|u_{\varepsilon}\right| \geq \frac{1}{2}$ in $\Omega$ as $\varepsilon \rightarrow 0$.

Theorem 7 is an improvement of [7, Theorem 6 in Appendix], where the boundary data satisfies the additional condition $\left|g_{\varepsilon}\right| \leq 1, \Omega$ is a square and $N_{\varepsilon} \ll|\log \varepsilon|$. We will follow the strategy in [7, generalizing to Lipschitz domains and general boundary data $g_{\varepsilon}: \partial \Omega \rightarrow \mathbb{R}^{2}$ with $N_{\varepsilon}$ satisfying the wider regime (4). The proof of Theorem 7 is divided into three parts:

Part 1 of the proof of Theorem 7 . We prove the following upper bound of $\left|u_{\varepsilon}\right|$ in $\Omega$ :

$$
\left\|u_{\varepsilon}\right\|_{L^{\infty}(\Omega)}-1 \lesssim \sqrt{\varepsilon N_{\varepsilon}} .
$$

For that, we start by denoting $\zeta=\left(1-\left|g_{\varepsilon}\right|\right)^{2}$ on $\partial \Omega$. The Cauchy-Schwarz inequality yields: ${ }^{6}$

$$
\frac{1}{2}\left|\partial_{\tau} g_{\varepsilon}\right|^{2}+\frac{1}{4 \varepsilon^{2}}\left(1-\left|g_{\varepsilon}\right|^{2}\right)^{2} \geq \frac{1}{8 \varepsilon^{2}} \zeta+\left(\frac{1}{8 \varepsilon^{2}} \zeta+\frac{1}{2}\left|\partial_{\tau}\right| g_{\varepsilon}||^{2}\right) \geq \frac{1}{8 \varepsilon^{2}} \zeta+\frac{1}{4 \varepsilon}\left|\partial_{\tau} \zeta\right| \quad \text { on } \partial \Omega \text {. }
$$

Using the embedding $W^{1,1}(\partial \Omega) \subset L^{\infty}(\partial \Omega)$, as $\mathcal{H}^{1}(\partial \Omega) \geq \varepsilon$, we deduce by (2):

$$
N_{\varepsilon}=\int_{\partial \Omega} \frac{1}{2}\left|\partial_{\tau} g_{\varepsilon}\right|^{2}+\frac{1}{4 \varepsilon^{2}}\left(1-\left|g_{\varepsilon}\right|^{2}\right)^{2} d \mathcal{H}^{1} \gtrsim \frac{1}{\varepsilon}\|\zeta\|_{L^{\infty}(\partial \Omega)}, \quad \text { as } \quad \varepsilon \rightarrow 0
$$

so that

$$
\delta_{\varepsilon}:=||\left|g_{\varepsilon}\right|-1 \|_{L^{\infty}(\partial \Omega)} \lesssim \sqrt{\varepsilon N_{\varepsilon}} .
$$

\footnotetext{
${ }^{6}$ For the more general energy [5], only the estimate $F(s) \gtrsim(1-s)^{2}$ is needed, which is a consequence of $(s-1) F^{\prime}(s) \gtrsim(1-s)^{2}$ and $F(1)=0$.
} 
Let $\tilde{\rho}_{\varepsilon}=1-\left|u_{\varepsilon}\right|^{2}$ in $\Omega$. Then (1) implies that

$$
-\Delta \tilde{\rho}_{\varepsilon}+\frac{2}{\varepsilon^{2}}\left|u_{\varepsilon}\right|^{2} \tilde{\rho}_{\varepsilon}=2\left|\nabla u_{\varepsilon}\right|^{2} \geq 0 \quad \text { in } \quad \Omega
$$

and $\tilde{\rho}_{\varepsilon}=1-\left|g_{\varepsilon}\right|^{2} \geq 1-\left(1+\delta_{\varepsilon}\right)^{2}$ on $\partial \Omega$. Thus, the maximum principl $\rrbracket^{7}$ implies that $\tilde{\rho}_{\varepsilon} \geq 1-\left(1+\delta_{\varepsilon}\right)^{2}$ in $\Omega$, i.e., $\left|u_{\varepsilon}\right| \leq 1+\delta_{\varepsilon}$ in $\Omega$ yielding (8) by (9).

Part 2 of the proof of Theorem 7 . We estimate a Hölder seminorm for $u_{\varepsilon}$.

Lemma 8 Let $\Omega \subset \mathbb{R}^{2}$ be a Lipschitz bounded domain. If $u_{\varepsilon}$ satisfies (1) and (4), then 8

$$
\left|u_{\varepsilon}(x)-u_{\varepsilon}(y)\right| \leq C\left(\frac{|x-y|}{\varepsilon}\right)^{\frac{1}{2}-} \quad \forall x, y \in \Omega,
$$

where $C>0$ depends only on the Lipschitz regularity of $\Omega$.

Remark 2 In general, we don't have that $\left\|\nabla u_{\varepsilon}\right\|_{L^{\infty}(\Omega)} \leq \frac{C}{\varepsilon}$ because this estimate can be violated by the boundary condition $g_{\varepsilon}$ on $\partial \Omega$. But since $g_{\varepsilon}$ belongs to $H^{1}(\partial \Omega)$ that embeds into the Hölder space $C^{0, \frac{1}{2}}(\partial \Omega)$, we can deduce an appropriate estimate of a Hölder seminorm for $u_{\varepsilon}$ in $\Omega$.

Proof of Lemma 8, Consider the rescaled map $\hat{u}(\hat{x})=u_{\varepsilon}(\varepsilon \hat{x})$ defined for $\hat{x} \in \Omega_{\varepsilon}=\varepsilon^{-1} \Omega$. (The map $\hat{u}$ depends on $\varepsilon$, we omit this dependence to simplify notation.) This map solves

$$
\left\{\begin{aligned}
-\Delta \hat{u} & =\left(1-|\hat{u}|^{2}\right) \hat{u} & & \text { in } \Omega_{\varepsilon}, \\
\hat{u} & =\hat{g} & & \text { on } \partial \Omega_{\varepsilon},
\end{aligned}\right.
$$

where $\hat{g}(\hat{x})=g_{\varepsilon}(\varepsilon \hat{x})$ for $\hat{x} \in \partial \Omega_{\varepsilon}$. We fix $x_{0} \in \Omega_{\varepsilon}$ and consider the Lipschitz domain

$$
\mathcal{R}=\mathcal{R}\left(x_{0}\right)=\frac{1}{\varepsilon}\left(\left(\varepsilon x_{0}+\varepsilon e^{i \gamma\left(\varepsilon x_{0}\right)} R\right) \cap \Omega\right),
$$

which is bi-Lipschitz homeomorphic to the unit ball $B$, with Lipschitz bounds uniform in $\varepsilon$ and $x_{0}$ and depending only on the Lipschitz regularity of $\Omega$, thanks to the definition of $R$, see (7). Since $|\hat{g}| \leq 1+\delta_{\varepsilon} \leq 2$ on $\partial \Omega_{\varepsilon}$ (by (9p) and $|\hat{u}| \leq 1+\delta_{\varepsilon} \leq 2$ in $\Omega_{\varepsilon}$ (by (8)) as $\varepsilon \rightarrow 0$, elliptic estimates in Lipschitz domains (see e.g. [12, 17, and [13, Section VI] for the theory of traces) yield

$$
\|\hat{u}\|_{H^{\frac{3}{2}-}(\mathcal{R})} \lesssim 1+\left\|\partial_{\tau} \hat{g}\right\|_{L^{2}\left(\partial \Omega_{\varepsilon}\right)} .
$$

The constant depends only on the Lipschitz regularity of the domain $\mathcal{R}$ (see e.g. the proof of Theorem 2 in [17]), and is therefore bounded independently of $x_{0} \in \Omega_{\varepsilon}$ and $\varepsilon \in(0,1]$. By Sobolev embedding we deduce that

$$
\|\hat{u}\|_{C^{0, \frac{1}{2}-}(\mathcal{R})} \lesssim 1+\left\|\partial_{\tau} \hat{g}\right\|_{L^{2}\left(\partial \Omega_{\varepsilon}\right)} \lesssim 1+\left(\varepsilon N_{\varepsilon}\right)^{\frac{1}{2}} .
$$

The constant in the Sobolev imbedding depends only on the Lipschitz regularity of $\Omega$, since the imbedding inequalities $\|v\|_{L^{4-}(B)} \lesssim\|v\|_{H^{\frac{1}{2}-}(B)}$ and $\|v\|_{C^{0, \frac{1}{2}-}(B)} \lesssim\|v\|_{W^{1,4-}(B)}$ are valid on the unit ball $B \subset \mathbb{R}^{2}$ and behave well under composition by a bi-Lipschitz homeomorphism. Since any

\footnotetext{
${ }^{7}$ This argument adapts to critical points of the general energy (5), provided $F^{\prime}(s) \geq 0$ for $s \geq 1$, see e.g. 15. Lemma 8.3].

${ }^{8}$ For the general energy (5) this argument only uses the fact that $F$ is $C^{1}$ and the validity of a uniform upper bound $\left\|u_{\varepsilon}\right\|_{\infty} \lesssim 1$, implied e.g. by 8 which is valid as soon as $F^{\prime}(s) \geq 0$ for $s \geq 1$.
} 
two points $x, y \in \Omega_{\varepsilon}$ which are close enough are contained in a domain $\mathcal{R}\left(x_{0}\right)$ for some $x_{0} \in \Omega_{\varepsilon}$, recalling once more that $|\hat{u}| \leq 2$ in $\Omega_{\varepsilon}$ (by $(8)$ ) we infer

$$
\|\hat{u}\|_{C^{0, \frac{1}{2}-}\left(\Omega_{\varepsilon}\right)} \lesssim 1+\left(\varepsilon N_{\varepsilon}\right)^{\frac{1}{2}} \lesssim 1 \quad \text { as } \quad \varepsilon \rightarrow 0 .
$$

The last inequality is due to our assumption (4). The conclusion follows by scaling back to $u_{\varepsilon}(x)=\hat{u}\left(\varepsilon^{-1} x\right)$.

Part 3 of the proof of Theorem 7. We start by estimating the normal derivative of $u_{\varepsilon}$ at the boundary $\partial \Omega$ :

Lemma 9 Let $\Omega \subset \mathbb{R}^{2}$ be a Lipschitz bounded domain. If $u_{\varepsilon}$ satisfies (1), then we have $9^{9}$

$$
\int_{\partial \Omega}\left|\partial_{\nu} u_{\varepsilon}\right|^{2} d \mathcal{H}^{1} \lesssim M_{\varepsilon}+N_{\varepsilon}
$$

Proof of Lemma 9. We use the Pohozaev identity for $u_{\varepsilon}$ in the spirit of [3, Proposition 3], the only difference is to adapt that result to the setting of Lipschitz domains $\Omega$. More precisely, we consider a map $V: \Omega \rightarrow \mathbb{R}^{2}$ that is $C^{1}$ in the closed domain $\bar{\Omega}$ and such that $V \cdot \nu \geq a>0$ on $\partial \Omega$ for some $a>0$ depending only on the Lipschitz regularity of $\Omega$ (see e.g. [8, Lemma 1.5.1.9]). Multiplying the equation (1) by $(V(x) \cdot \nabla) u_{\varepsilon}$ and integrating by parts, as $V \in C^{1}(\bar{\Omega})$, we deduce by (2) and (3):

$$
\begin{aligned}
\left|\frac{1}{\varepsilon^{2}} \int_{\Omega} u_{\varepsilon}\left(1-\left|u_{\varepsilon}\right|^{2}\right) \cdot(V(x) \cdot \nabla) u_{\varepsilon} d x\right|= & \mid \frac{1}{4 \varepsilon^{2}} \int_{\Omega} \nabla \cdot V\left(1-\left|u_{\varepsilon}\right|^{2}\right)^{2} d x \\
& -\frac{1}{4 \varepsilon^{2}} \int_{\partial \Omega} V(x) \cdot \nu\left(1-\left|g_{\varepsilon}\right|^{2}\right)^{2} d \mathcal{H}^{1} \mid \lesssim M_{\varepsilon}+N_{\varepsilon}, \quad(10) \\
\int_{\Omega} \Delta u_{\varepsilon} \cdot(V(x) \cdot \nabla) u_{\varepsilon} d x= & \int_{\partial \Omega}\left((\nu \cdot \nabla) u_{\varepsilon} \cdot(V \cdot \nabla) u_{\varepsilon}-\frac{1}{2} V \cdot \nu\left|\nabla u_{\varepsilon}\right|^{2}\right) d \mathcal{H}^{1} \\
& +\int_{\Omega}\left(\frac{1}{2} \nabla \cdot V\left|\nabla u_{\varepsilon}\right|^{2}-\sum_{j=1,2} \partial_{j} u_{\varepsilon} \cdot\left(\partial_{j} V \cdot \nabla\right) u_{\varepsilon}\right) d x .
\end{aligned}
$$

For $x \in \partial \Omega$, we decompose $V=s(x) \nu+t(x) \tau$ where $s, t \in L^{\infty}(\partial \Omega), s(x)=V \cdot \nu \geq a>0$ for a.e. $x \in \partial \Omega$, and $\nabla u_{\varepsilon}=\nu \otimes \partial_{\nu} u_{\varepsilon}+\tau \otimes \partial_{\tau} g_{\varepsilon}$ on $\partial \Omega$. By (1), (2), (3), (10) and (11), as $V \in C^{1}(\bar{\Omega})$, we conclude by Young's inequality:

$$
M_{\varepsilon}+N_{\varepsilon} \gtrsim \int_{\partial \Omega}\left(\frac{s(x)}{2}\left|\partial_{\nu} u_{\varepsilon}\right|^{2}-\frac{s(x)}{2}\left|\partial_{\tau} g_{\varepsilon}\right|^{2}+t(x) \partial_{\nu} u_{\varepsilon} \cdot \partial_{\tau} g_{\varepsilon}\right) d \mathcal{H}^{1} \gtrsim \int_{\partial \Omega}\left|\partial_{\nu} u_{\varepsilon}\right|^{2} d \mathcal{H}^{1}-N_{\varepsilon} .
$$

We use Lemma 9 to prove the following estimate of the potential energy in small balls (of radius $\left.\ll \varepsilon^{\alpha}\right)$. To simplify notation, we denote the energy density by

$$
e_{\varepsilon}\left(u_{\varepsilon}\right):=\frac{1}{2}\left|\nabla u_{\varepsilon}\right|^{2}+\frac{1}{4 \varepsilon^{2}}\left(1-\left|u_{\varepsilon}\right|^{2}\right)^{2}, \quad u_{\varepsilon}: \Omega \rightarrow \mathbb{R}^{2} .
$$

(In the context of the energy (5), only the assumption $F \in C^{1}$ is needed for the following estimate).

\footnotetext{
${ }^{9}$ In the context of the general energy [5], we need only the assumption that $F \in C^{1}$.
} 
Lemma 10 Let $\Omega \subset \mathbb{R}^{2}$ be a Lipschitz bounded domain and $u_{\varepsilon}$ be a solution of (1) in the regime (4). Fix $1>\alpha_{1}>\alpha_{2}>\alpha>0$. There exists $C \geq 1$ such that for every $x_{0} \in \Omega$, we can find $r_{0}=r_{0}\left(x_{0}\right) \in\left(\varepsilon^{\alpha_{1}}, \varepsilon^{\alpha_{2}}\right)$ such that

$$
\int_{\partial\left(B\left(x_{0}, r_{0}\right) \cap \Omega\right)} e_{\varepsilon}\left(u_{\varepsilon}\right) d \mathcal{H}^{1} \leq \frac{C\left(1+M_{\varepsilon}\right)}{r_{0}|\log \varepsilon|}
$$

for every $\varepsilon \leq \varepsilon_{0}$ with $\varepsilon_{0}=\varepsilon_{0}\left(\alpha_{2}, \alpha\right)>0$. Moreover, we have that

$$
\frac{1}{\varepsilon^{2}} \int_{B\left(x_{0}, r_{0}\right) \cap \Omega}\left(1-\left|u_{\varepsilon}\right|^{2}\right)^{2} d x \leq \frac{\tilde{C}\left(1+M_{\varepsilon}\right)}{|\log \varepsilon|}
$$

for some $\tilde{C} \geq 1$.

Proof of Lemma 10. We distinguish two steps:

Step 1. Proof of $(12)$. Assume by contradiction that for every $C \geq 1$ there exists $x \in \Omega$ such that for every $r \in\left(\varepsilon^{\alpha_{1}}, \varepsilon^{\alpha_{2}}\right)$ we have

$$
\int_{\partial(B(x, r) \cap \Omega)} e_{\varepsilon}\left(u_{\varepsilon}\right) d \mathcal{H}^{1} \geq \frac{C\left(1+M_{\varepsilon}\right)}{r|\log \varepsilon|} .
$$

Since $N_{\varepsilon} \varepsilon^{\alpha} \ll 1$, by $(2)$ and Lemma 9 , there exists $c_{1}>0$ such that

$$
\int_{\partial \Omega} e_{\varepsilon}\left(u_{\varepsilon}\right) d \mathcal{H}^{1} \leq c_{1}\left(M_{\varepsilon}+N_{\varepsilon}\right) \leq \frac{1+M_{\varepsilon}}{2 \varepsilon^{\alpha_{2}}|\log \varepsilon|} \leq \frac{C\left(1+M_{\varepsilon}\right)}{2 r|\log \varepsilon|}, \quad \forall r \in\left(\varepsilon^{\alpha_{1}}, \varepsilon^{\alpha_{2}}\right)
$$

for every $\varepsilon \leq \varepsilon_{0}$ (with $\varepsilon_{0}>0$ depending on $\alpha_{2}$ and $\alpha$ ). Therefore, we deduce that

$$
\int_{\partial B(x, r) \cap \Omega} e_{\varepsilon}\left(u_{\varepsilon}\right) d \mathcal{H}^{1} \geq \frac{C\left(1+M_{\varepsilon}\right)}{2 r|\log \varepsilon|} .
$$

Integrating in $r \in\left(\varepsilon^{\alpha_{1}}, \varepsilon^{\alpha_{2}}\right)$, we obtain by $[3]$ :

$M_{\varepsilon}=\int_{\Omega} e_{\varepsilon}\left(u_{\varepsilon}\right) d x \geq \int_{B\left(x, \varepsilon^{\alpha_{2}}\right) \cap \Omega} e_{\varepsilon}\left(u_{\varepsilon}\right) d x \geq \int_{\varepsilon^{\alpha_{1}}}^{\varepsilon^{\alpha_{2}}} d r \int_{\partial B(x, r) \cap \Omega} e_{\varepsilon}\left(u_{\varepsilon}\right) d \mathcal{H}^{1} \geq \frac{C\left(\alpha_{1}-\alpha_{2}\right)\left(1+M_{\varepsilon}\right)}{2}$

which is a contradiction with the fact that $C$ can be arbitrary large.

Step 2. Proof of (13). Let $\nu$ be the outer unit normal vector at the boundary of the domain

$$
\mathcal{D}:=B\left(x_{0}, r_{0}\right) \cap \Omega .
$$

As in the proof of Lemma 9, we use the Pohozaev identity for the solution $u_{\varepsilon}$ of (1). Indeed, multiplying the equation by $\left(x-x_{0}\right) \cdot \nabla u_{\varepsilon}$ and integrating by parts, we deduce:

$$
\begin{aligned}
\int_{\mathcal{D}}-\Delta u_{\varepsilon} \cdot\left(\left(x-x_{0}\right) \cdot \nabla u_{\varepsilon}\right) d x= & \int_{\partial \mathcal{D}}\left(\frac{1}{2}\left(x-x_{0}\right) \cdot \nu\left|\nabla u_{\varepsilon}\right|^{2}-\partial_{\nu} u_{\varepsilon} \cdot\left(\left(x-x_{0}\right) \cdot \nabla\right) u_{\varepsilon}\right) d \mathcal{H}^{1}, \\
\frac{1}{\varepsilon^{2}} \int_{\mathcal{D}} u_{\varepsilon}\left(1-\left|u_{\varepsilon}\right|^{2}\right) \cdot\left(\left(x-x_{0}\right) \cdot \nabla u_{\varepsilon}\right) d x= & \frac{1}{2 \varepsilon^{2}} \int_{\mathcal{D}}\left(1-\left|u_{\varepsilon}\right|^{2}\right)^{2} d x \\
& -\frac{1}{4 \varepsilon^{2}} \int_{\partial \mathcal{D}}\left(x-x_{0}\right) \cdot \nu\left(1-\left|u_{\varepsilon}\right|^{2}\right)^{2} d \mathcal{H}^{1} .
\end{aligned}
$$

Since $\left|x-x_{0}\right| \leq r_{0}$ on $\partial \mathcal{D}$, by $[12$, we deduce that 13$]$ holds true.

The conclusion of Theorem 7 comes from the following result: 
Lemma 11 Let $\Omega \subset \mathbb{R}^{2}$ be a Lipschitz bounded domain. If $u_{\varepsilon}$ satisfies (1) and (4), then we have 10

$$
\left\|\left|u_{\varepsilon}\right|^{2}-1\right\|_{L^{\infty}(\Omega)} \lesssim\left(\frac{1+M_{\varepsilon}}{|\log \varepsilon|}\right)^{\frac{1}{6}-} .
$$

Proof. Let $x_{0} \in \Omega$ and set $1>A \geq 0$ such that

$$
4 C A^{\frac{1}{2}-}=\frac{\left.|1-| u_{\varepsilon}\left(x_{0}\right)\right|^{2} \mid}{2},
$$

where $C \geq 1$ is given by Lemma 8 . By Lemma 8 , we obtain for any $y \in B\left(x_{0}, A \varepsilon\right) \cap \Omega$

$$
\left.|1-| u_{\varepsilon}(y)\right|^{2}|\geq| 1-\left|u_{\varepsilon}\left(x_{0}\right)\right|^{2} \mid-4 C A^{\frac{1}{2}-}=\frac{\left.|1-| u_{\varepsilon}\left(x_{0}\right)\right|^{2} \mid}{2}
$$

as $\left|u_{\varepsilon}(y)\right|+\left|u_{\varepsilon}\left(x_{0}\right)\right| \leq 4$. Hence, for small $\varepsilon$,

$$
\begin{aligned}
\int_{B\left(x_{0}, A \varepsilon\right) \cap \Omega}\left(1-\left|u_{\varepsilon}(y)\right|^{2}\right)^{2} d y & \geq C(\Omega) A^{2} \varepsilon^{2}\left(1-\left|u_{\varepsilon}\left(x_{0}\right)\right|^{2}\right)^{2} \\
& \geq \tilde{C}(\Omega) \varepsilon^{2}\left(1-\left|u_{\varepsilon}\left(x_{0}\right)\right|^{2}\right)^{6+},
\end{aligned}
$$

where $C(\Omega), \tilde{C}(\Omega)>0$. We have that $B\left(x_{0}, A \varepsilon\right) \subset B\left(x_{0}, r_{0}\right)$ for $\varepsilon \leq \varepsilon_{0}$ with $\varepsilon_{0}$ depending only on $\alpha_{1}$ in Lemma 10 . Thus, by (13), we obtain

$$
\left(1-\left|u_{\varepsilon}\left(x_{0}\right)\right|^{2}\right)^{6+} \leq \hat{C} \frac{1+M_{\varepsilon}}{|\log \varepsilon|}
$$

and the conclusion follows.

\section{Proof of Theorem 1}

The main idea is to improve the convergence of $\left|u_{\varepsilon}\right|$ to 1 locally in $L^{2}$-norm; this involves improving the local estimate of the potential energy (13) to a positive power of $\varepsilon$ and then the argument in Lemma 11 yields the conclusion (i.e., the desired estimate in $L^{\infty}$-norm of $\left|u_{\varepsilon}\right|-1$ in our main result).

Let $x_{0} \in \Omega$ and $\varepsilon>0$. By Fubini's theorem we may choose $t \in[1 / 2,1]$ such that the domain

$$
\mathcal{R}=\mathcal{R}_{t}\left(x_{0}\right)
$$

defined in (7) satisfies

$$
\int_{\partial \mathcal{R} \cap \Omega} \frac{1}{2}\left|\nabla u_{\varepsilon}\right|^{2}+\frac{1}{4 \varepsilon^{2}}\left(1-\left|u_{\varepsilon}\right|^{2}\right)^{2} d \mathcal{H}^{1} \lesssim M_{\varepsilon}
$$

Recall that $\mathcal{R}$ is bi-Lipschitz homeomorphic to the unit ball $B$, in particular it is simply connected. Moreover by Theorem 7 if $\kappa$ is small enough then $u_{\varepsilon}$ does not vanish. So we may write

$$
u_{\varepsilon}=\rho_{\varepsilon} e^{i \varphi_{\varepsilon}} \quad \text { in } \mathcal{R},
$$

with $\rho_{\varepsilon}, \varphi_{\varepsilon} \in H^{1}(\mathcal{R})$ (moreover, $\rho_{\varepsilon}^{2}$ and $\varphi_{\varepsilon}$ are smooth in $\mathcal{R}$ as $u_{\varepsilon}$ is smooth by standard elliptic regularity). The system (1) writes in terms of $\rho_{\varepsilon}$ and $\varphi_{\varepsilon}$ :

$$
\left\{\begin{array}{l}
-\Delta \rho_{\varepsilon}+\rho_{\varepsilon}\left|\nabla \varphi_{\varepsilon}\right|^{2}=\frac{1}{\varepsilon^{2}} \rho_{\varepsilon}\left(1-\rho_{\varepsilon}^{2}\right) \quad \text { in } \mathcal{R} . \\
\nabla \cdot\left(\rho_{\varepsilon}^{2} \nabla \varphi_{\varepsilon}\right)=0
\end{array}\right.
$$

\footnotetext{
${ }^{10}$ For the general energy 5 we only need here $(s-1)^{2} \lesssim F(s)$.
} 
Step 1. We prove the following estimate ${ }^{11}$ of $\nabla \varphi_{\varepsilon}$ in $L^{q}(\mathcal{R})$, where $q=4-$ :

$$
\left\|\nabla \varphi_{\varepsilon}\right\|_{L^{q}(\mathcal{R})} \lesssim 1+N_{\varepsilon}^{\frac{1}{2}}+M_{\varepsilon}^{\frac{1}{2}} .
$$

Indeed, by (2), (9), Lemma 9 and (16), we note that

$$
\begin{aligned}
& \int_{\partial \Omega \cap \mathcal{R}}\left|\nabla \varphi_{\varepsilon}\right|^{2} d \mathcal{H}^{1} \lesssim \int_{\partial \Omega}\left|\nabla u_{\varepsilon}\right|^{2} d \mathcal{H}^{1} \lesssim N_{\varepsilon}+M_{\varepsilon} \\
& \text { and } \int_{\Omega \cap \partial \mathcal{R}}\left|\nabla \varphi_{\varepsilon}\right|^{2} d \mathcal{H}^{1} \lesssim \int_{\partial \mathcal{R} \cap \Omega}\left|\nabla u_{\varepsilon}\right|^{2} d \mathcal{H}^{1} \lesssim M_{\varepsilon} .
\end{aligned}
$$

Therefore, by the Poincaré-Wirtinger inequality, up to adding a constant to $\varphi_{\varepsilon}$, we can assume that

$$
\left\|\varphi_{\varepsilon}\right\|_{H^{1}(\partial \mathcal{R})} \lesssim 1+N_{\varepsilon}^{\frac{1}{2}}+M_{\varepsilon}^{\frac{1}{2}}
$$

By the theory of traces in Lipschitz domains (see e.g. [13, Section VI.2]), for $s=1-$ there is a continuous extension operator from $H^{s}(\partial \mathcal{R})$ to $H^{s+1 / 2}(\mathcal{R})$, and its operator norm is bounded by a constant depending only on the Lipschitz regularity of $\mathcal{R}$, hence only on the Lipschitz regularity of $\Omega$. Thus there exists an extension $\Phi \in H^{\frac{3}{2}-}(\mathcal{R})$ of $\left.\varphi_{\varepsilon}\right|_{\partial \mathcal{R}}$ such that

$$
\|\Phi\|_{H^{\frac{3}{2}-}(\mathcal{R})} \lesssim 1+N_{\varepsilon}^{\frac{1}{2}}+M_{\varepsilon}^{\frac{1}{2}}
$$

By Sobolev embedding $H^{\frac{1}{2}-}(\mathcal{R}) \subset L^{4-}(\mathcal{R})$ we deduce the bound

$$
\|\nabla \Phi\|_{L^{q}(\mathcal{R})} \lesssim 1+N_{\varepsilon}^{\frac{1}{2}}+M_{\varepsilon}^{\frac{1}{2}}
$$

The constant in the Sobolev embedding depends only on the Lipschitz regularity of $\Omega$ since $\mathcal{R}$ is bi-Lipschitz homeomorphic to the unit ball (with Lipschitz constants depending only on the Lipschitz regularity of $\Omega$ ). Denoting

$$
\psi:=\varphi_{\varepsilon}-\Phi \in H_{0}^{1}(\mathcal{R})
$$

by 17$), \psi$ solves

$$
\Delta \psi=\nabla \cdot\left(\left(1-\rho_{\varepsilon}^{2}\right) \nabla \varphi_{\varepsilon}-\nabla \Phi\right) \quad \text { in } \quad \mathcal{R}
$$

so that elliptic estimates in Lipschitz domains (see e.g. [12, Theorem 0.5] or [17, Theorem 2]) yield

$$
\left\|\nabla \varphi_{\varepsilon}\right\|_{L^{q}(\mathcal{R})} \leq C\left(1+\left\|\left(1-\rho_{\varepsilon}^{2}\right) \nabla \varphi_{\varepsilon}\right\|_{L^{q}(\mathcal{R})}+\|\nabla \Phi\|_{L^{q}(\mathcal{R})}\right) .
$$

By Theorem 7. $C\left|1-\rho_{\varepsilon}^{2}\right| \leq \frac{1}{2}$ in $\mathcal{R}$ for $\kappa>0$ small enough. This implies

$$
\left\|\nabla \varphi_{\varepsilon}\right\|_{L^{q}(\mathcal{R})} \lesssim 1+\|\nabla \Phi\|_{L^{q}(\mathcal{R})} .
$$

The last term can be estimated by (21) and this proves 18 .

Step 2. An improved local estimate of the potential energy. We will prove the following:

Lemma 12 Let $\Omega \subset \mathbb{R}^{2}$ be a Lipschitz bounded domain. If $u_{\varepsilon}$ satisfies (1) and (4), then

$$
\frac{1}{\varepsilon^{2}} \int_{\mathcal{R}}\left(1-\left|u_{\varepsilon}\right|^{2}\right)^{2} d x \lesssim \varepsilon^{1-}\left(1+N_{\varepsilon}+M_{\varepsilon}\right)\left(1+M_{\varepsilon}\right)^{\frac{1}{2}-},
$$

for every point $x_{0} \in \Omega$ with the associated domain $\mathcal{R}$ in 15 .

\footnotetext{
${ }^{11}$ For the general energy [5], no modification is required for this step since the equation satisfied by $\varphi_{\varepsilon}$ stays the same.
} 
Proof. Multiplying (17) by $1-\rho_{\varepsilon}^{2}$, as $\rho_{\varepsilon} \geq 1 / 2$ in $\mathcal{R}$ (by Theorem 7), integration by parts yields ${ }^{12}$

$$
\begin{aligned}
\frac{1}{2 \varepsilon^{2}} \int_{\mathcal{R}}\left(1-\rho_{\varepsilon}^{2}\right)^{2} d x & \leq \frac{1}{\varepsilon^{2}} \int_{\mathcal{R}} \rho_{\varepsilon}\left(1-\rho_{\varepsilon}^{2}\right)^{2} d x \\
& =-\int_{\mathcal{R}}\left(1-\rho_{\varepsilon}^{2}\right) \Delta \rho_{\varepsilon} d x+\int_{\mathcal{R}} \rho_{\varepsilon}\left(1-\rho_{\varepsilon}^{2}\right)\left|\nabla \varphi_{\varepsilon}\right|^{2} d x \\
& =-\int_{\partial \mathcal{R}}\left(1-\rho_{\varepsilon}^{2}\right) \partial_{\nu} \rho_{\varepsilon} d \mathcal{H}^{1}-2 \int_{\mathcal{R}} \rho_{\varepsilon}\left|\nabla \rho_{\varepsilon}\right|^{2} d x+\int_{\mathcal{R}} \rho_{\varepsilon}\left(1-\rho_{\varepsilon}^{2}\right)\left|\nabla \varphi_{\varepsilon}\right|^{2} d x \\
& \leq\left\|1-\rho_{\varepsilon}^{2}\right\|_{L^{2}(\partial \mathcal{R})}\left\|\partial_{\nu} \rho_{\varepsilon}\right\|_{L^{2}(\partial \mathcal{R})}+2\left\|\nabla \varphi_{\varepsilon}\right\|_{L^{q}(\mathcal{R})}^{2}\left\|1-\rho_{\varepsilon}^{2}\right\|_{L^{\frac{q}{q-2}}(\mathcal{R})} \\
& \lesssim \varepsilon\left(M_{\varepsilon}+N_{\varepsilon}\right)+\varepsilon^{1-}\left(1+N_{\varepsilon}+M_{\varepsilon}\right) M_{\varepsilon}^{\frac{1}{2}-}
\end{aligned}
$$

for $q=4-$, where we used

- (2) and (16) yielding $\left\|1-\rho_{\varepsilon}^{2}\right\|_{L^{2}(\partial \mathcal{R})} \lesssim \varepsilon\left(M_{\varepsilon}+N_{\varepsilon}\right)^{\frac{1}{2}}$;

- 19p yielding $\left\|\partial_{\nu} \rho_{\varepsilon}\right\|_{L^{2}(\partial \mathcal{R})} \lesssim\left(M_{\varepsilon}+N_{\varepsilon}\right)^{\frac{1}{2}}$;

- 18 and the interpolation inequality for $\lambda=\frac{2(q-2)}{q}=1-$

$$
\left\|1-\rho_{\varepsilon}^{2}\right\|_{L^{\frac{q}{q-2}(\mathcal{R})}} \leq\left\|1-\rho_{\varepsilon}^{2}\right\|_{L^{\infty}(\mathcal{R})}^{1-\lambda}\left\|1-\rho_{\varepsilon}^{2}\right\|_{L^{2}(\mathcal{R})}^{\lambda} \stackrel{\sqrt[3]{3,}, 8]}{\lesssim} \varepsilon^{\lambda} M_{\varepsilon}^{\frac{\lambda}{2}}
$$

yielding the last estimate.

Step 3. Conclusion of Theorem 1. Applying the arguments in the proof of Lemma 11 in the domain $\mathcal{R}=\mathcal{R}_{t}\left(x_{0}\right)$ defined at 15$)$, we find

$$
\left(\left|u_{\varepsilon}\left(x_{0}\right)\right|^{2}-1\right)^{6+} \lesssim \frac{1}{\varepsilon^{2}} \int_{\mathcal{R}}\left(1-\rho_{\varepsilon}^{2}\right)^{2} d x \lesssim \varepsilon^{1-}\left(1+N_{\varepsilon}+M_{\varepsilon}\right)\left(1+M_{\varepsilon}\right)^{\frac{1}{2}-} .
$$

The last inequality follows from the previous step. Since $x_{0} \in \Omega$ is arbitrary and the constant depends only on the Lipschitz regularity of $\Omega$, this proves Theorem 1 .

\section{Optimality of the regime (4)}

In this section, we prove Propositions 2 to 5

Proof of Proposition 2, Let $\Omega$ be a cone of opening angle $\theta_{0}$ and height 1, see Figure 4. Consider the point $P_{\varepsilon}$ on the medial axis at distance $s_{\varepsilon}$ from the corner, where

$$
s_{\varepsilon}=\varepsilon^{\mu} \quad \text { with } 0<\mu<1 .
$$

Set $\alpha=\frac{1+\mu}{2} \in(0,1)$. We also denote by $d_{\varepsilon}$ the distance of $P_{\varepsilon}$ to the boundary $\partial \Omega$. For $\theta_{1}=\theta_{0}+\eta$ (where, possibly lowering $\eta$, we may assume $\eta<\theta_{0}$ ) consider the cone $K_{1}$ of opening $\theta_{1}$ and height 1 centered at $P_{\varepsilon}$ and with the same medial axis. The boundaries of the two cones intersect in two points at a distance $r_{\varepsilon}$ from $P_{\varepsilon}$. It follows that $\Omega \subset B\left(P_{\varepsilon}, r_{\varepsilon}\right) \cup K_{1}\left(\right.$ as $\left.s_{\varepsilon}<r_{\varepsilon}\right)$,

$$
d_{\varepsilon}=s_{\varepsilon} \sin \frac{\theta_{0}}{2} \sim \varepsilon^{\mu} \quad \text { and } \quad r_{\varepsilon}=s_{\varepsilon} \frac{\sin \frac{\theta_{0}}{2}}{\sin \frac{\eta}{2}} \sim \varepsilon^{\mu} .
$$

\footnotetext{
${ }^{12}$ For the general energy (5), this estimate holds thanks to the assumption $(s-1) F^{\prime}(s) \gtrsim(s-1)^{2}$ for $s \geq 0$.
} 
Figure 2: The cones $\Omega$ and $K_{1}$ of opening angles $\theta_{0}$ and $\theta_{1}$ respectively.

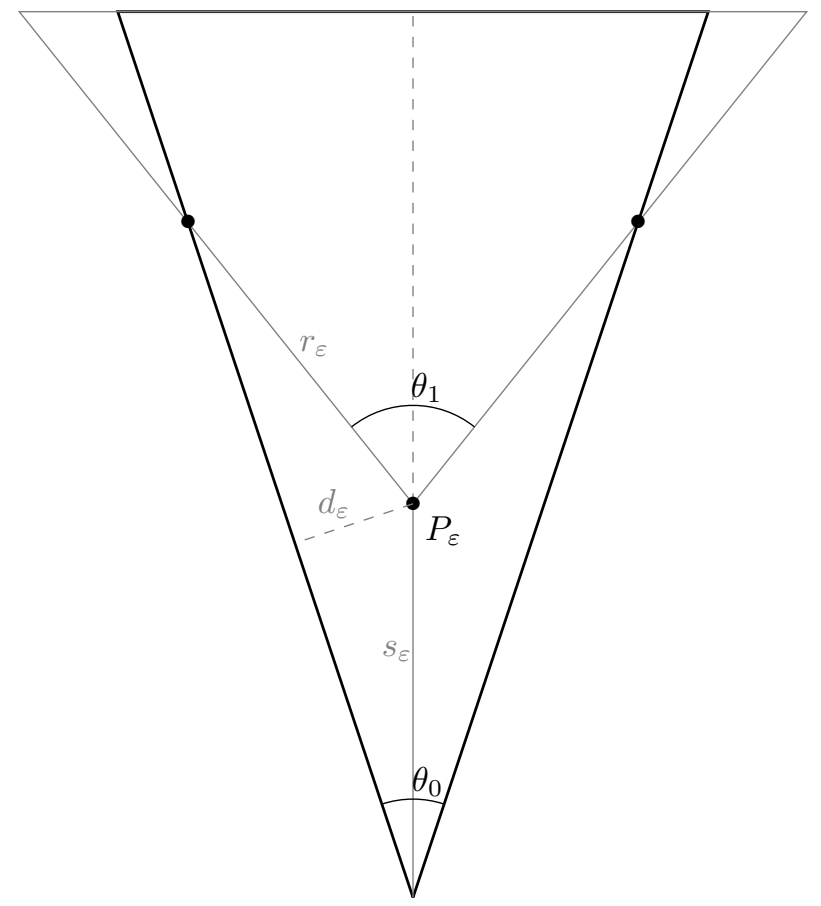

We consider the following degree-one vortex solution $u_{\varepsilon}$ of $(1)$ :

$$
u_{\varepsilon}(x)=f\left(\frac{\left|x-P_{\varepsilon}\right|}{\varepsilon}\right) \frac{x-P_{\varepsilon}}{\left|x-P_{\varepsilon}\right|} \quad \text { for every } \quad x \in \mathbb{R}^{2},
$$

where $P_{\varepsilon}$ is the vortex point (i.e., $\left.u_{\varepsilon}\left(P_{\varepsilon}\right)=0\right), f:[0, \infty) \rightarrow[0,1)$ is the smooth radial profile given by the unique solution of

$$
-f^{\prime \prime}-\frac{1}{r} f^{\prime}+\frac{1}{r^{2}} f=f\left(1-f^{2}\right) \quad \text { for every } \quad r \in(0, \infty),
$$

with $f(0)=0$ and $\lim _{r \rightarrow \infty} f(r)=1 ; f$ and $f^{\prime}$ have the following asymptotics for $r \rightarrow \infty$ (see [6, 9])

$$
f(r)=1-\frac{1}{2 r^{2}}-\frac{9}{8 r^{4}}+O\left(r^{-6}\right), \quad f^{\prime}(r)=\frac{1}{r^{3}}+\frac{9}{2 r^{5}}+O\left(r^{-7}\right) .
$$

In a point $x \in \mathbb{R}^{2}$ with $\left|x-P_{\varepsilon}\right|=t$, the Ginzburg-Landau energy density is given by

$$
e_{\varepsilon}\left(u_{\varepsilon}(x)\right)=\frac{1}{2}\left(\frac{\left|f^{\prime}\left(\frac{t}{\varepsilon}\right)\right|^{2}}{\varepsilon^{2}}+\frac{\left|f\left(\frac{t}{\varepsilon}\right)\right|^{2}}{t^{2}}\right)+\frac{1}{4 \varepsilon^{2}}\left(1-\left|f\left(\frac{t}{\varepsilon}\right)\right|^{2}\right)^{2},
$$

so that for $t \geq \varepsilon$, we find

$$
e_{\varepsilon}\left(u_{\varepsilon}(x)\right)=\frac{1}{2 t^{2}}+\frac{1}{\varepsilon^{2}} O\left(\frac{\varepsilon^{4}}{t^{4}}\right)
$$

Recalling that $r_{\varepsilon} \gg \varepsilon$, we obtain by integrating over $K_{1} \backslash B\left(P_{\varepsilon}, r_{\varepsilon}\right)$ :

$$
\int_{K_{1} \backslash B\left(P_{\varepsilon}, r_{\varepsilon}\right)} e_{\varepsilon}\left(u_{\varepsilon}\right) d x \leq \theta_{1} \int_{r_{\varepsilon}}^{2} t\left(\frac{1}{2 t^{2}}+\frac{1}{\varepsilon^{2}} O\left(\frac{\varepsilon^{4}}{t^{4}}\right)\right) d t \leq \frac{\theta_{1}}{2} \log \frac{2}{r_{\varepsilon}}+O\left(\frac{\varepsilon^{2}}{r_{\varepsilon}^{2}}\right) .
$$


In $B\left(P_{\varepsilon}, r_{\varepsilon}\right)$, using 222 and the fact that $f(0)=0$ and $\left|f^{\prime}\right| \lesssim 1$ (in particular, $|f(t)| \lesssim t$ for $t>0$ ), we estimate

$$
\begin{aligned}
\int_{B\left(P_{\varepsilon}, r_{\varepsilon}\right)} e_{\varepsilon}\left(u_{\varepsilon}\right) d x & \leq \pi\left(\int_{0}^{\varepsilon}+\int_{\varepsilon}^{r_{\varepsilon}}\right)\left[\left(\frac{\left|f^{\prime}\left(\frac{t}{\varepsilon}\right)\right|^{2}}{\varepsilon^{2}}+\frac{\left|f\left(\frac{t}{\varepsilon}\right)\right|^{2}}{t^{2}}\right)+\frac{1}{2 \varepsilon^{2}}\left(1-\left|f\left(\frac{t}{\varepsilon}\right)\right|^{2}\right)^{2}\right] t d t \\
& \leq C \int_{0}^{\varepsilon} \frac{t}{\varepsilon^{2}} d t+\pi \log \frac{r_{\varepsilon}}{\varepsilon}+O(1)=\pi \log \frac{r_{\varepsilon}}{\varepsilon}+O(1) .
\end{aligned}
$$

As $\Omega \subset B\left(P_{\varepsilon}, r_{\varepsilon}\right) \cup K_{1}$, it follows that the interior energy $M_{\varepsilon}$ is estimated as:

$$
M_{\varepsilon}=\int_{\Omega} e_{\varepsilon}\left(u_{\varepsilon}\right) d x \leq \pi \log \frac{r_{\varepsilon}}{\varepsilon}+\frac{\theta_{1}}{2} \log \frac{2}{r_{\varepsilon}}+O(1) \leq\left(\pi(1-\mu)+\frac{\theta_{1}}{2} \mu\right)|\log \varepsilon|+C
$$

where $C>0$ is a constant depending only on $\eta$ and $\theta_{0}$. Note that for $\mu$ sufficiently close to 1 and $\varepsilon$ small enough, this implies

$$
M_{\varepsilon} \leq\left(\frac{\theta_{0}}{2}+\eta\right)|\log \varepsilon|
$$

To estimate the boundary energy $N_{\varepsilon}$, we write $\partial \Omega=\Gamma_{1} \cup \Gamma_{2}^{+} \cup \Gamma_{2}^{-}$, where $\Gamma_{1}$ is the basis of the cone, and $\Gamma_{2}^{ \pm}$are the two sides of the cone adjacent to its vertex. Since $P_{\varepsilon}$ is at distance $\sim 1$ of $\Gamma_{1}$, it holds

$$
\int_{\Gamma_{1}} e_{\varepsilon}\left(u_{\varepsilon}\right) d \mathcal{H}^{1}=O(1)
$$

On the rest of the boundary, note that for every point $x \in \Gamma_{2}^{ \pm}$that has a distance $s$ from the orthogonal projections of $P_{\varepsilon}$ onto $\Gamma_{2}^{ \pm}$, we have

$$
e_{\varepsilon}\left(u_{\varepsilon}(x)\right)=\frac{1}{2 t^{2}}+\frac{1}{\varepsilon^{2}} O\left(\frac{\varepsilon^{4}}{t^{4}}\right), \quad \text { where } t=\left|x-P_{\varepsilon}\right|=\sqrt{s^{2}+d_{\varepsilon}^{2}},
$$

since $t \geq d_{\varepsilon} \sim \varepsilon^{\mu} \gg \varepsilon$. We can thus estimate

$$
N_{\varepsilon} \leq 2 \int_{-\infty}^{\infty}\left(\frac{1}{2\left(s^{2}+d_{\varepsilon}^{2}\right)}+C \frac{\varepsilon^{2}}{\left(s^{2}+d_{\varepsilon}^{2}\right)^{2}}\right) d s+O(1) \lesssim \frac{1}{d_{\varepsilon}}+\frac{\varepsilon^{2}}{d_{\varepsilon}^{3}} \lesssim \frac{1}{s_{\varepsilon}} \sim \frac{1}{\varepsilon^{\mu}} \ll \frac{1}{\varepsilon^{\alpha}}
$$

as $\alpha$ was chosen such that $\alpha=\frac{1+\mu}{2}<1$. So (4) holds with $\kappa=\frac{\theta_{0}}{2}+\eta$, while $u_{\varepsilon}\left(P_{\varepsilon}\right)=0$.

Remark 3 Applying the construction in the proof of Proposition 2 to a half-space domain, we deduce that a necessary condition in order that Theorem 1 holds true is given by $\kappa \leq \frac{\pi}{2}$ in (4) (even for smooth domains $\Omega$ ).

Proof of Proposition 3. Let $f:[0, \infty) \rightarrow[0,1]$ be a smooth function with $f(0)=0, f(r)=1$ for $r \geq 1$ and $\left|f^{\prime}(r)\right| \leq C$. Let $x_{0} \in \partial \Omega$ and consider $v_{\varepsilon}(x)=f\left(\frac{x-x_{0}}{\varepsilon}\right)$ for every $x \in \mathbb{R}^{2}$. Let $g_{\varepsilon}=$ $\left(v_{\varepsilon}, 0\right)$ on $\partial \Omega$ and let $u_{\varepsilon}$ be a minimizer of the Ginzburg-Landau energy with Dirichlet boundary conditions $g_{\varepsilon}$, in particular, $u_{\varepsilon}\left(x_{0}\right)=g_{\varepsilon}\left(x_{0}\right)=0$. Then $u_{\varepsilon}$ satisfies (1) and (by minimality) $M_{\varepsilon} \leq E_{\varepsilon}\left(v_{\varepsilon} ; \Omega\right) \lesssim 1$ while $N_{\varepsilon} \lesssim \frac{1}{\varepsilon}$.

Proof of Proposition 4. Since $\Omega$ is smooth, bounded and simply connected, upon applying a conformal diffeomorphism we assume $\Omega=B(0,1)$ is the unit disk ${ }^{13}$ Our example is strongly inspired by [1, Example 1] and [2, Lemma 4.1]. For $\eta>0$ small we consider

$$
v_{\eta}(z)=\frac{z-(1-\eta)}{1-(1-\eta) z} \quad \text { for } z \in B(0,1) \subset \mathbb{C},
$$

\footnotetext{
13 Only the bulk energy changes and is bounded (up to a multiplicative constant depending on $\Omega$ ) by the new bulk energy in the disk $B(0,1)$.
} 
and denote by $g_{\eta}$ its boundary datum $g_{\eta}(\theta)=v_{\eta}\left(e^{i \theta}\right)$, which satisfies $\left|g_{\eta}\right|=1$ on $\partial B(0,1)$. As explained in [2, Lemma 4.1], the map $v_{\eta}$ satisfies

$$
\frac{1}{2} \int_{B(0,1)}\left|\nabla v_{\eta}\right|^{2} d x=\pi, \quad \text { and } \operatorname{deg}\left(v_{\eta}, \partial B(0,1)\right)=1 .
$$

Lengthy but direct computations show that for $\theta \in(-\pi, \pi)$ we have

$$
\left|g_{\eta}^{\prime}(\theta)\right|^{2}=\frac{(2-\eta)^{2}}{\eta^{2}} \frac{1}{\left(1+2 \frac{1-\eta}{\eta^{2}}(1-\cos \theta)\right)^{2}} .
$$

Moreover $1-\cos \theta \geq \frac{2}{\pi^{2}} \theta^{2}$ for $\theta \in(-\pi, \pi)$, and therefore, for small $\eta$,

$$
\begin{aligned}
\int_{-\pi}^{\pi}\left|g_{\eta}^{\prime}(\theta)\right|^{2} d \theta & \lesssim \frac{1}{\eta^{2}} \int_{-\pi}^{\pi} \frac{d \theta}{\left(1+\frac{\theta^{2}}{\eta^{2}}\right)^{2}}=\frac{1}{\eta} \int_{-\frac{\pi}{\eta}}^{\frac{\pi}{\eta}} \frac{d t}{\left(1+t^{2}\right)^{2}} \\
& \lesssim \frac{1}{\eta} .
\end{aligned}
$$

For $0<r<1$ and $\theta \in(-\pi, \pi)$, setting $a=1-\eta$ we have

$$
1-\left|v_{\eta}\left(r e^{i \theta}\right)\right|^{2}=\left(1-a^{2}\right) \frac{1-r^{2}}{\left(1+a^{2} r^{2}-2 a r \cos \theta\right)} .
$$

In order to estimate $\int\left(1-\left|v_{\eta}\right|^{2}\right)^{2}$ we first compute

$$
I:=\int_{-\pi}^{\pi} \frac{d \theta}{\left(1+a^{2} r^{2}-2 a r \cos \theta\right)^{2}} .
$$

The change of variable $x=\tan \frac{\theta}{2}$ gives $d \theta=2 d x /\left(1+x^{2}\right)$ and $\cos \theta=\left(1-x^{2}\right) /\left(1+x^{2}\right)$, so

$$
\begin{aligned}
I & =2 \int_{-\infty}^{\infty} \frac{1+x^{2}}{\left((1-a r)^{2}+(1+a r)^{2} x^{2}\right)^{2}} d x \\
& =\frac{2}{(1+a r)^{4}} \int_{-\infty}^{\infty} \frac{1+x^{2}}{\left(A^{2}+x^{2}\right)^{2}} d x, \quad A:=\frac{1-a r}{1+a r} .
\end{aligned}
$$

We have

$$
\begin{aligned}
\int_{-\infty}^{\infty} \frac{1+x^{2}}{\left(A^{2}+x^{2}\right)^{2}} d x & =\int_{-\infty}^{\infty} \frac{d x}{A^{2}+x^{2}}+\left(1-A^{2}\right) \int_{-\infty}^{\infty} \frac{d x}{\left(A^{2}+x^{2}\right)^{2}} \\
& =\frac{1}{A} \int_{-\infty}^{\infty} \frac{d t}{1+t^{2}}+\left(1-A^{2}\right) \frac{1}{A^{3}} \int_{-\infty}^{\infty} \frac{d t}{\left(1+t^{2}\right)^{2}} \\
& =\frac{1}{A}\left(\pi+\frac{1-A^{2}}{A^{2}} \frac{\pi}{2}\right)=\frac{\pi}{2} \frac{1+A^{2}}{A^{3}}
\end{aligned}
$$

Since $1+a r \geq 1$, plugging this back into $I$ yields

$$
I=2 \pi \frac{1+a^{2} r^{2}}{(1+a r)^{3}} \frac{1}{(1-a r)^{3}} \leq \frac{2 \pi}{(1-a r)^{3}} .
$$


We deduce

$$
\begin{aligned}
\int_{B(0,1)}\left(1-\left|v_{\eta}\right|^{2}\right)^{2} d x & =\left(1-a^{2}\right)^{2} \int_{0}^{1}\left(1-r^{2}\right)^{2} I r d r \\
& \lesssim \eta^{2} \int_{0}^{1} \frac{(1-r)^{2}}{(1-a r)^{3}} d r=\eta^{2} \int_{0}^{1} \frac{t^{2}}{(a t+\eta)^{3}} d t \\
& =\frac{1}{\eta} \int_{0}^{1} \frac{t^{2}}{\left(\frac{a}{\eta} t+1\right)^{3}} d t=\frac{\eta^{2}}{a^{3}} \int_{0}^{\frac{a}{\eta}} \frac{s^{2}}{(s+1)^{3}} d s \\
& \lesssim \eta^{2} \log \frac{1}{\eta}
\end{aligned}
$$

In particular,

- choosing $\eta=\varepsilon /|\log \varepsilon|^{\frac{1}{2}}$ and $u_{\varepsilon}$ minimizing the Ginzburg-Landau energy with Dirichlet condition $u_{\varepsilon}=g_{\eta}$ on $\partial B(0,1)$, we have that $u_{\varepsilon}$ is of modulus one and degree one on $\partial B(0,1)$ while $M_{\varepsilon} \lesssim 1$ and $N_{\varepsilon} \lesssim \frac{|\log \varepsilon|^{\frac{1}{2}}}{\varepsilon}$

- choosing $\eta:=\frac{\varepsilon \sqrt{\kappa}}{L}$ with $L>0$ big enough and $u_{\varepsilon}$ minimizing the Ginzburg-Landau energy with Dirichlet condition $u_{\varepsilon}=g_{\eta}$ on $\partial B(0,1)$, we have that $u_{\varepsilon}$ is of modulus one and degree one on $\partial B(0,1)$ while $M_{\varepsilon} \leq \kappa|\log \varepsilon|$ and $N_{\varepsilon} \lesssim \frac{1}{\varepsilon}$.

Proof of Proposition 5. Upon locally flattening the boundary and rescaling the domain (thus only introducing multiplicative constants in all estimates), we may assume that $\Omega$ is as in Example 1, i.e. it contains the upper half unit ball:

$$
\Omega \cap B(0,1)=\left\{x=\left(x_{1}, x_{2}\right) \in B(0,1): x_{2}>0\right\} .
$$

As in Example 1, for $\eta=\eta(\varepsilon) \ll \varepsilon$ we consider the boundary data $g_{\varepsilon}: \partial \Omega \rightarrow \mathbb{S}^{1}$ such that $g_{\varepsilon}(x)=e^{i \phi_{\varepsilon}}$ with

$$
\phi_{\varepsilon}(x)= \begin{cases}0 & \text { if } x \in \partial \Omega \backslash B(0, \eta), \\ \pi\left(1-\frac{\left|x_{1}\right|}{\eta}\right) & \text { if } x=\left(x_{1}, x_{2}\right) \in \partial \Omega \cap B(0, \eta) .\end{cases}
$$

As in Example 1 we then have that $N_{\varepsilon} \sim \frac{1}{\eta}$ and any map $u_{\varepsilon}$ minimizing $E_{\varepsilon}(\cdot ; \Omega)$ with Dirichlet boundary data $u_{\varepsilon}=g_{\varepsilon}$ on $\partial \Omega$ satisfies $M_{\varepsilon} \lesssim 1$.

It remains to show that

$$
\left.\sup _{\Omega}|1-| u_{\varepsilon}\right|^{2} \mid \geq \frac{1}{2} \quad \text { for } \varepsilon \ll 1 .
$$

The idea is to use the decomposition $u_{\varepsilon}=v_{\varepsilon}+w_{\varepsilon}$ with

$$
\left\{\begin{array} { r l r l } 
{ - \Delta v _ { \varepsilon } } & { = \frac { 1 } { \varepsilon ^ { 2 } } ( 1 - | u _ { \varepsilon } | ^ { 2 } ) u _ { \varepsilon } } & { } & { \text { in } \Omega , } \\
{ v _ { \varepsilon } } & { = 0 } & { } & { \text { on } \partial \Omega , }
\end{array} \quad \text { and } \quad \left\{\begin{array}{rlrl}
\Delta w_{\varepsilon}=0 & \text { in } \Omega, \\
w_{\varepsilon}=g_{\varepsilon} & \text { on } \partial \Omega,
\end{array}\right.\right.
$$

(see also the proof of Lemma 3 in [7]).

The maximum principle can be used to see that $\left|w_{\varepsilon}\right| \leq 1$ and $\left|u_{\varepsilon}\right| \leq 1$ in $\Omega$. Indeed, since $-\Delta\left|w_{\varepsilon}\right|^{2}=-2\left|\nabla w_{\varepsilon}\right|^{2} \leq 0$ in $\Omega$ and $\left|w_{\varepsilon}\right|=1$ on $\partial \Omega$, the maximal principle implies that $\left|w_{\varepsilon}\right| \leq 1$ in $\Omega$. Moreover, recalling that $\tilde{\rho}_{\varepsilon}=1-\left|u_{\varepsilon}\right|^{2}$ satisfies

$$
-\Delta \tilde{\rho}_{\varepsilon}+\frac{2}{\varepsilon^{2}}\left|u_{\varepsilon}\right|^{2} \tilde{\rho}_{\varepsilon}=2\left|\nabla u_{\varepsilon}\right|^{2} \geq 0 \quad \text { in } \quad \Omega,
$$


and $\tilde{\rho}_{\varepsilon}=0$ on $\partial \Omega$, the maximum principle ensures that $\tilde{\rho}_{\varepsilon} \geq 0$ in $\Omega$, i.e. $\left|u_{\varepsilon}\right| \leq 1$ in $\Omega$.

We deduce that $\left|v_{\varepsilon}\right| \leq 2$ in $\Omega$. Using this together with the equation satisfied by $v_{\varepsilon}$, and the interpolation inequality 3 ]

$$
\left\|\nabla v_{\varepsilon}\right\|_{L^{\infty}(\Omega)} \lesssim\left\|v_{\varepsilon}\right\|_{L^{\infty}(\Omega)}^{\frac{1}{2}}\left\|\Delta v_{\varepsilon}\right\|_{L^{\infty}(\Omega)}^{\frac{1}{2}},
$$

we find that $\left\|\nabla v_{\varepsilon}\right\|_{L^{\infty}(\Omega)} \lesssim \frac{1}{\varepsilon}$. In particular, since $v_{\varepsilon}=0$ on $\partial \Omega$, we deduce that $\left|v_{\varepsilon}\right| \lesssim \frac{\eta}{\varepsilon} \ll 1$ in $\Omega \cap B(0,2 \eta)$. As a consequence we have

$$
\left.\sup _{\Omega \cap B(0,2 \eta)}|| u_{\varepsilon}\right|^{2}-\left|w_{\varepsilon}\right|^{2} \mid \ll 1,
$$

and to prove 23 it suffices to show that

$$
\left|w_{\varepsilon}(0, \eta)\right|^{2}<\frac{1}{2} \quad \text { for } \varepsilon \ll 1 .
$$

To this end we rescale $w_{\varepsilon}$, setting $\tilde{w}_{\eta}(x)=w_{\varepsilon}(\eta x)$ so that

$$
\begin{aligned}
& \begin{cases}\Delta \tilde{w}_{\eta}=0 & \text { in } B\left(0, \frac{1}{\eta}\right) \cap\left\{x_{2}>0\right\}, \\
\tilde{w}_{\eta}=e^{i \tilde{\phi}} & \text { on } B\left(0, \frac{1}{\eta}\right) \cap\left\{x_{2}=0\right\},\end{cases} \\
& \text { where } \tilde{\phi}\left(x_{1}, 0\right)= \begin{cases}0 & \text { if }\left|x_{1}\right|>1, \\
\pi\left(1-\left|x_{1}\right|\right) & \text { if }\left|x_{1}\right| \leq 1 .\end{cases}
\end{aligned}
$$

Since $\left|\tilde{w}_{\eta}\right| \leq 1$ and $\tilde{\phi}$ is Lipschitz, elliptic estimates ensure that $\tilde{w}_{\eta}$ is bounded in $C_{l o c}^{0, \alpha}\left(\left\{x_{2} \geq 0\right\}\right)$ for any $\alpha \in(0,1)$ and therefore admits a subsequence converging locally uniformly to a map $\tilde{w}_{0}:\left\{x_{2} \geq 0\right\} \rightarrow \mathbb{C}$ which solves

$$
\left\{\begin{aligned}
\Delta \tilde{w}_{0}=0 & \text { in }\left\{x_{2}>0\right\}, \\
\tilde{w}_{0}=e^{i \tilde{\phi}} & \text { on }\left\{x_{2}=0\right\},
\end{aligned}\right.
$$

and satisfies $\left|\tilde{w}_{0}\right| \leq 1$. This system has a unique bounded solution, given by the Poisson formula

$$
\tilde{w}_{0}\left(x_{1}, x_{2}\right)=\frac{x_{2}}{\pi} \int_{-\infty}^{\infty} \frac{e^{i \tilde{\phi}(t, 0)}}{\left(x_{1}-t\right)^{2}+x_{2}^{2}} d t .
$$

In particular no subsequence is needed for the locally uniform convergence, and we have

$$
w_{\varepsilon}(0, \eta)=\tilde{w}_{\eta}(0,1) \longrightarrow \tilde{w}_{0}(0,1) \quad \text { as } \varepsilon \rightarrow 0 .
$$

Using the explicit expression of $\tilde{\phi}$ we compute

$$
\begin{aligned}
\tilde{w}_{0}(0,1) & =\frac{2}{\pi}\left(\int_{0}^{1} \frac{e^{i \pi(1-t)}}{1+t^{2}} d t+\int_{1}^{\infty} \frac{1}{1+t^{2}} d t\right) \\
& =\frac{2}{\pi} \int_{0}^{1} \frac{1-e^{-i \pi t}}{1+t^{2}} d t .
\end{aligned}
$$

It can be checked that

$$
\left|\int_{0}^{1} \frac{1-e^{-i \pi t}}{1+t^{2}} d t\right| \leq 1
$$

and we infer that

$$
\lim _{\varepsilon \rightarrow 0}\left|w_{\varepsilon}(0, \eta)\right|^{2} \leq \frac{4}{\pi^{2}}<\frac{1}{2},
$$

which implies 24). 


\section{Acknowledgements}

We thank Petru Mironescu, Roger Moser and Luc Nguyen for interesting comments. R.I. acknowledges partial support by the ANR project ANR-14-CE25-0009-01. X.L. acknowledges partial support by the ANR project ANR-18-CE40-0023.

\section{References}

[1] Berlyand, L., And Mironescu, P. Ginzburg-Landau minimizers with prescribed degrees. Capacity of the domain and emergence of vortices. J. Funct. Anal. 239, 1 (2006), 76-99.

[2] Berlyand, L., And Mironescu, P. Ginzburg-Landau minimizers in perforated domains with prescribed degrees. hal-00747687, June 2008.

[3] Bethuel, F., Brezis, H., And HÉlein, F. Asymptotics for the minimization of a GinzburgLandau functional. Calc. Var. Partial Differential Equations 1, 2 (1993), 123-148.

[4] Bethuel, F., Brezis, H., And Hélein, F. Ginzburg-Landau vortices. Progress in Nonlinear Differential Equations and their Applications, 13. Birkhäuser Boston Inc., Boston, MA, 1994.

[5] Bethuel, F., Orlandi, G., And Smets, D. Improved estimates for the Ginzburg-Landau equation: the elliptic case. Ann. Sc. Norm. Super. Pisa Cl. Sci. (5) 4, 2 (2005), 319-355.

[6] Chen, X., Elliott, C. M., And QI, T. Shooting method for vortex solutions of a complexvalued Ginzburg-Landau equation. Proc. Roy. Soc. Edinburgh Sect. A 124, 6 (1994), 10751088.

[7] Côte, R., Ignat, R., And Miot, E. A thin-film limit in the Landau-Lifshitz-Gilbert equation relevant for the formation of Néel walls. J. Fixed Point Theory Appl. 15, 1 (2014), $241-272$.

[8] Grisvard, P. Elliptic problems in nonsmooth domains, vol. 69 of Classics in Applied Mathematics. Society for Industrial and Applied Mathematics (SIAM), Philadelphia, PA, 2011.

[9] Hervé, R.-M., AND Hervé, M. Étude qualitative des solutions réelles d'une équation différentielle liée à l'équation de Ginzburg-Landau. Ann. Inst. H. Poincaré Anal. Non Linéaire 11, 4 (1994), 427-440.

[10] Ignat, R., And Kurzke, M. Global Jacobian and $\Gamma$-convergence in a Ginzburg-Landau model for boundary vortices. arXiv:1910.06039, 2019.

[11] Ignat, R., And Otto, F. A compactness result for Landau state in thin-film micromagnetics. Ann. Inst. H. Poincaré Anal. Non Linéaire 28, 2 (2011), 247-282.

[12] Jerison, D., And Kenig, C. E. The inhomogeneous Dirichlet problem in Lipschitz domains. J. Funct. Anal. 130, 1 (1995), 161-219.

[13] Jonsson, A., And Wallin, H. Function spaces on subsets of $\mathbf{R}^{n}$. Math. Rep. 2, 1 (1984), xiv +221 .

[14] Kunzke, M. Boundary vortices in thin magnetic films. Calc. Var. Partial Differential Equations 26, 1 (2006), 1-28.

[15] Lamy, X. Bifurcation analysis in a frustrated nematic cell. J. Nonlinear Sci. 24, 6 (2014), $1197-1230$. 
[16] Sandier, E., And Serfaty, S. Vortices in the magnetic Ginzburg-Landau model, vol. 70 of Progress in Nonlinear Differential Equations and their Applications. Birkhäuser Boston, Inc., Boston, MA, 2007.

[17] Savaré, G. Regularity results for elliptic equations in Lipschitz domains. J. Funct. Anal. 152, 1 (1998), 176-201. 